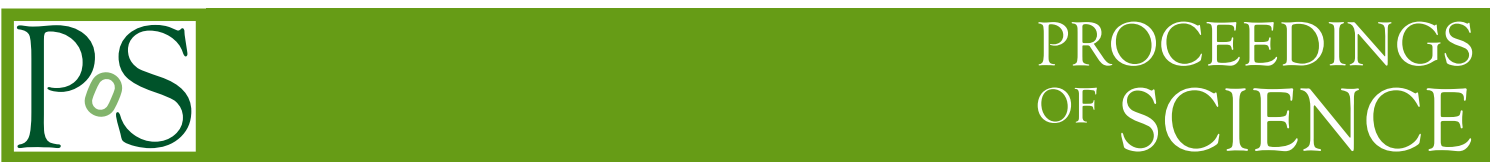

\title{
Exploring the QCD Phase Diagram
}

\author{
Volker Koch* \\ Nuclear Science Division, Lawrence Berkeley National Laboratory, Berkeley, CA 94720, USA \\ E-mail: vkochelbl.gov
}

\begin{abstract}
In this contribution we will address a few selected topics concerning the search for a phase structure in the QCD phase diagram. We will extract some constraints for model calculations from basic consideration about the QCD phase diagram. We will also discuss to what extent lattice calculations may be compared to measurements, and what the necessary corrections will be. Finally we will address the dynamics associated with the spinodal break up due to a first order phase transition.
\end{abstract}

9th International Workshop on Critical Point and Onset of Deconfinement - CPOD2014,

17-21 November 2014

ZiF (Center of Interdisciplinary Research), University of Bielefeld, Germany

\footnotetext{
* Speaker.
} 


\section{Introduction}

The search for a phase structure in the QCD phase diagram has been at the center of strong interaction research for many years and has recently received renewed interest with the start of a dedicated program at RHIC, the so called RHIC beam energy scan (BES). The major motivation for the BES is to experimentally map the QCD phase diagram by varying the collision energy from $\sqrt{s_{N N}}=200 \mathrm{GeV}$ down to $\sqrt{s_{N N}}=7.7 \mathrm{GeV}$ in order to create systems of ever higher net-baryon density. Lattice QCD calculations have found that at vanishing net-baryon density or chemical potential, $\mu_{B}$, the transition from a gas of hadrons to a quark gluon plasma (QGP) is an analytic cross-over [1,2] which takes place at a pseudo-critical temperature of $T_{p c}=154 \pm 9 \mathrm{MeV}[3,4,5]$. In contrast, at large net-baryon density the transition to a QGP may very well be of first order as several model calculations predict (see e.g. [6]). If this were the case, the first order co-existence region will have to end in a critical point at some finite density and temperature. Unfortunately due the fermion sign problem, Lattice QCD calculations have only a limited reach in the chemical potential, $\mu_{B} / T \lesssim 3$. While there are potential hints for a critical point in exploratory lattice calculations $[7,8,9]$, no definitive evidence has been provided yet. Therefore, a systematic experimental search for the critical point (CP) and a first order phase co-existence region is required.

In the following we will discuss selected aspects of the theoretical and experimental exploration of the QCD phase diagram. We will begin with a discussion of general properties of the QCD phase diagram and how those provide constraints for model calculations. Next, we will discuss fluctuation measurements and why they are sensitive to the phase structure. In particular we emphasize various corrections necessary for a meaningful comparison with theoretical calculations. Finally, we will turn our discussion to the first order co-existence region. In particular we will discuss to which extent the existence of the co-existence region and its associated unstable (spinodal) region can be explored in experiment.

\section{The QCD phase diagram: General remarks}

Let us start our discussion with a few general observations about the QCD phase diagram. This discussion will be very qualitative and, therefore, we will label the high density QCD phase simply as "QGP" and will not further specify what it's detailed microscopic structure is. All that is relevant for our discussion is that at high density and sufficiently low temperature, there is coexistence between nuclear matter and this new phase.

The top two panels of Fig. 1 show the $(T, \mu)$ phase diagram of a typical liquid-gas phase diagram on the left, and on the right what is commonly shown as the QCD phase diagram. Aside from the scale, which has been omitted on purpose, both look qualitatively the same. However, this is not the case if we plot the phase structure in a pressure-temperature $(P, T)$ diagram. In this case the liquid-gas first order transition line starts at the origin, i.e. at $(P, T)=(0,0)$ indicating that for a liquid-gas system at vanishing temperature we have co-existence between the dense (liquid) phase and the vacuum. This is why water droplets (or atomic nuclei) are stable. In case of the QCD transition, the situation is quite different. At zero temperature the high density "QGP"-phase does not co-exist with the vacuum but rather with high density nuclear matter. Consequently, the co-existence pressure at $T=0$ is finite and large. Indeed, given a nuclear matter equation of state 

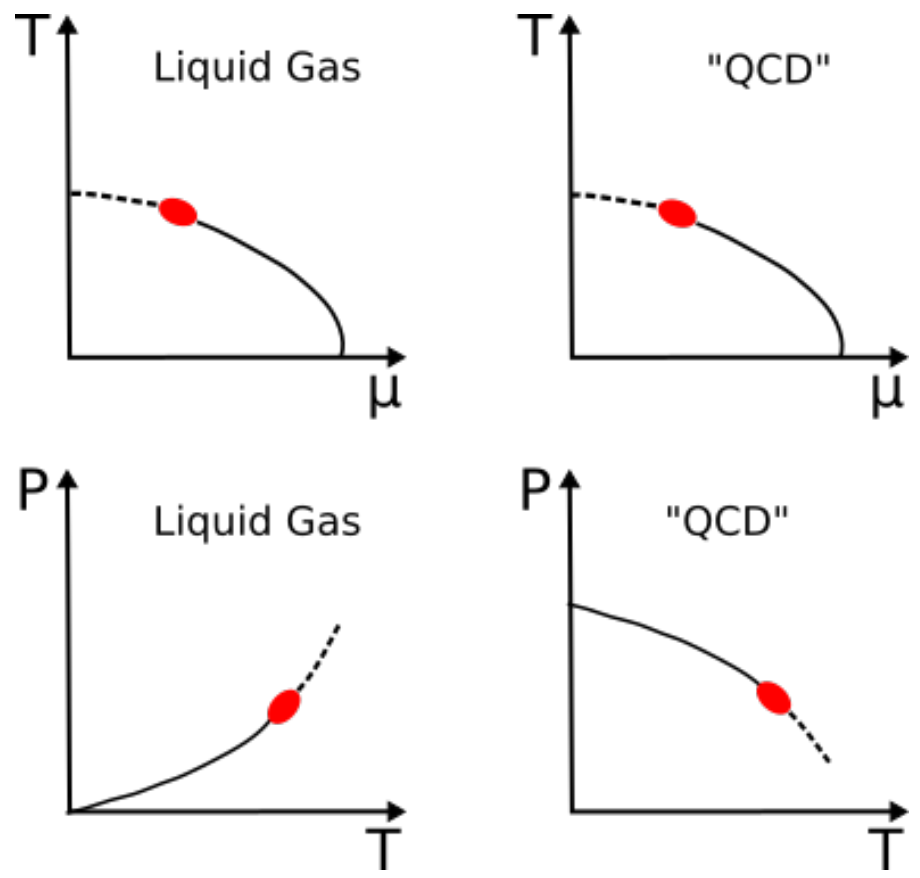

Figure 1: Schematic comparison of a typical liquid-gas transition (left panels) and the QCD transition (right panels). The upper row shows the familiar $(\mu, T)$ diagrams while the lower row shows them in the variables $(T, P)$. Here we have assumed that for the QCD phase transition the co-existence pressure at vanishing temperature is larger than then the pressure at the pseudo-critical transition at vanishing net-baryon density.

$[10,11]$, which is able to account for the recently discovered two solar mass neutron stars, the pressure of cold nuclear matter at about 2.5 times the ground state density is as high as the pressure obtained from lattice QCD at the transition for vanishing chemical potential, $P\left(\rho=2.5 \rho_{0}, T=\right.$ $0) \simeq P\left(\rho=0, T=T_{p c}\right)$. Therefore, if the zero temperature transition happens at densities larger than $\rho \gtrsim 2.5 \rho_{0}$ the pressure along the co-existence line will decrease with increasing temperature, contrary to the liquid-gas case. This difference in slope of the pressure along the co-existence line has some interesting consequences [12]. Using the Clausius-Clapeyron relation for co-existent phases 1 and 2

$$
\frac{d P}{d T}=\frac{S_{1} / B_{1}-S_{2} / B_{2}}{1 / \rho_{1}-1 / \rho_{2}}
$$

and assuming that phase 2 is always the dense phase, so that $\left(1 / \rho_{1}-1 / \rho_{2}\right)>0$, we find that in case of a liquid-gas transition, where $d P / d T>$, the entropy per baryon is larger in the gas phase then in the liquid (high density) phase,

$$
\left(\frac{S}{B}\right)_{\text {gas }}>\left(\frac{S}{B}\right)_{\text {liquid }}
$$

For the QCD transition, on the other hand, the dense phase will most likely have a larger entropy 
per baryon ${ }^{1}$

$$
\left(\frac{S}{B}\right)_{\text {hadron-gas }}<\left(\frac{S}{B}\right)_{Q G P} \text {. }
$$

As a consequence, for the QCD transition a hydrodynamic trajectory, which follows a constant entropy to baryon-number ratio, will be moving towards the critical point after it hits the co-existence line from the right. For a liquid-gas transition, on the other hand, it will be moving away from the CP. Below we will discuss further consequences of the facts that co-existence pressure at vanishing temperature is finite and large, and that the entropy per baryon in the dense phase is larger than in the dilute phase.

Let us stress again that the QCD transition can not be of the liquid-gas type in the sense that there is no phase co-existence between the vacuum and the QGP. Otherwise, one would be able to observe QGP droplets in a detector. While this may be obvious, it should be pointed out that most effective models which predict a critical point are of the liquid-gas type and predict co-existence with the vacuum and the QGP. (See e.g. the results of the Polyakov-Quark meson model shown in Fig. 3). Therefore, the phenomenological value of these models is rather limited.

After these more general observations about the QCD phase diagram let us next discuss how one can explore the phase diagram in experiment and potential issues related to this endeavor.

\section{Exploring the phase diagram in experiment}

In order to search for possible structures in the phase diagram one needs to (a) scan the various regions of the phase diagram and (b) have suitable observables which are sensitive to changes in the phase structure. Scanning the phase diagram is achieved by varying the beam energy; with decreasing collision energy the landing point in the phase diagram moves to higher values for the chemical potential and lower temperature. This is the central idea of the RHIC beam energy scan. Concerning observables, there have been many suggestions ranging from flow to HBT measurements. Most attention, however, has been payed to ratios of cumulants of distributions of conserved charges, in particular the net-baryon number cumulants $[13,14]$. The reason why cumulants are sensitive to phase changes can be easily understood from the following simple argument: Any structure in the phase diagram is ultimately related with variations (or singularities in the derivatives) of the free energy (or pressure). For example, crossing the co-existence line along a line of constant temperature, the density, i.e. the first derivative of the pressure with respect to the chemical potential, is discontinuous. In a small system these discontinuities are washed out leaving a more smooth transition. However, if we were able to measure ever higher derivatives of the pressure with respect to the chemical potentials we would be able to identify this transition. Thus, the question remains how to measure derivatives with respect to the chemical potential. The answer to this is surprisingly simple: Cumulants of the baryon number are nothing but these derivatives, as can be seen from their definition

$$
C_{B}^{n} \equiv \frac{d^{n}}{d(\mu / T)^{n}} \ln Z=\frac{d^{n}}{d(\mu / T)^{n}} \frac{P V}{T} .
$$

\footnotetext{
${ }^{1}$ This assumes that the zero temperature transition happens at densities $\rho>2.5 \rho_{0}$.
} 
As long as we stay in the same phase, the pressure and density vary smoothly, and thus the cumulants, i.e. derivatives, are small and well behaved. Only in the region close to the phase-transition do we expect large derivatives or cumulants. At high beam energies we will spend most of the time in the QGP phase and at very low energies we will only be in the hadronic phase. In both cases the cumulants will be small. However, somewhere in between we will hit the phase-transition and the cumulants will be large. Consequently, we expect a non-monotonic behavior of the cumulants as a function of beam energy, with a maximum of the cumulant ratio somewhere near the critical point or co-existence region, as first suggested in [15] and later refined in [13, 14].

Meanwhile cumulants of the net-proton distribution have been measured by the STAR collaboration [16], and a recent update with increased acceptance has been reported at this conference [17]. The most recent data show an intriguing increase of the cumulant ratio $C^{4} / C^{2}$ towards the lowest energies accessible by the BES, albeit with significant error bars. While this result is very interesting and encouraging, one should not forget other, more mundane, sources of fluctuations. One of the most obvious is the fluctuations induced by the stopping of the nuclei. After all, the only way to increase the net-baryon density in a heavy ion collision is to stop the baryons from the incoming nuclei. Clearly, the amount of nucleons stopped varies from collision to collision even for a fixed impact parameter, and it is not evident that these stopping fluctuations are similar to the thermal particle number fluctuations in a grand canonical ensemble. Thus an independent assessment of these "stopping fluctuations" is absolutely necessary. For example, one could analyze the co-variance $\left\langle\delta N_{p}(y>0) \delta N_{p}(y<0)\right\rangle$ of the number of protons at forward, $y>0$, and backward, $y<0$, rapidities.

Another issue which needs to be understood is the dependence of the cumulant ratio on the experimental acceptance. Indeed, the recent analysis by the STAR collaboration shows that the cumulant ratio, in particular the enhancement at low energies, depends on the acceptance both in transverse momentum and rapidity (see Fig. 4 in [17]). This is not unexpected and has been discussed in [18] using a simple binomial model for the acceptance. However, this finding has important implications for any comparison of both model and lattice QCD calculations with the measured data. Lattice QCD and most model calculations of cumulant ratios determine the cumulants in the infinite volume limit. Consequently, these calculations capture all the correlations. In an experiment, however, one has to deal with a finite acceptance, and it is, therefore, not clear a priori if all the correlations are captured by the measurement. To illustrate this point consider a simple two-particle correlation function in rapidity $y$ :

$$
\left\langle n\left(y_{1}\right)\left(n\left(y_{2}\right)-\delta\left(y_{1}-y_{2}\right)\right)\right\rangle \equiv\left\langle n\left(y_{1}\right)\right\rangle\left\langle n\left(y_{2}\right)\right\rangle\left(1+C\left(y_{1}, y_{2}\right)\right)
$$

with

$$
C\left(y_{1}, y_{2}\right)=C_{0} \exp \left(\frac{-\left(y_{1}-y_{2}\right)^{2}}{2 \sigma^{2}}\right)
$$

so that

$$
\frac{\left\langle(\delta N)^{2}\right\rangle}{\langle N\rangle}=1+\langle N\rangle \int_{-\Delta / 2}^{\Delta / 2} d y_{1} d y_{2} C\left(y_{1}, y_{2}\right) .
$$


The resulting scaled variance $\left\langle(\delta N)^{2}\right\rangle /\langle N\rangle$ is shown in Fig. 2 as a function of the acceptance $\Delta$ divided by the correlation length $\sigma$ (blue line). Clearly, the acceptance has to be much larger than the correlation length, $\Delta / \sigma \simeq 10$, in order reproduce the correct scaled variance. It is this asymptotic value which would have to be compared with lattice calculations. However, things get even more complicated. Lattice QCD works in the grand-canonical ensemble where, contrary to a heavy ion collision, charges are only conserved on the average, i.e. there is no explicit conservation of the global charge. Obviously, global charge conservation affects fluctuations of conserved charges since in the limit of $100 \%$ acceptance the fluctuations will vanish. Even for a finite acceptance, global charge conservation modify the fluctuations as discussed in [19]. To illustrate this effect, we have used the simple leading order formula of [20] to account for charge conservation noting that a more complete treatment has been derived in [21]. The resulting scaled variances are shown as the magenta, yellow and green lines, where we have assumed that the total charge is distributed over an interval of of $\Delta_{\text {charge }} / \sigma=5,10,20$, respectively. Obviously, things become rather tricky now. Looking, for example, at the green and yellow curves, charge conservation together with a positive correlation may conspire to give a cumulant ratio of approximately unity, which usually would indicate Poisson statistics, i.e. absence of any correlation.

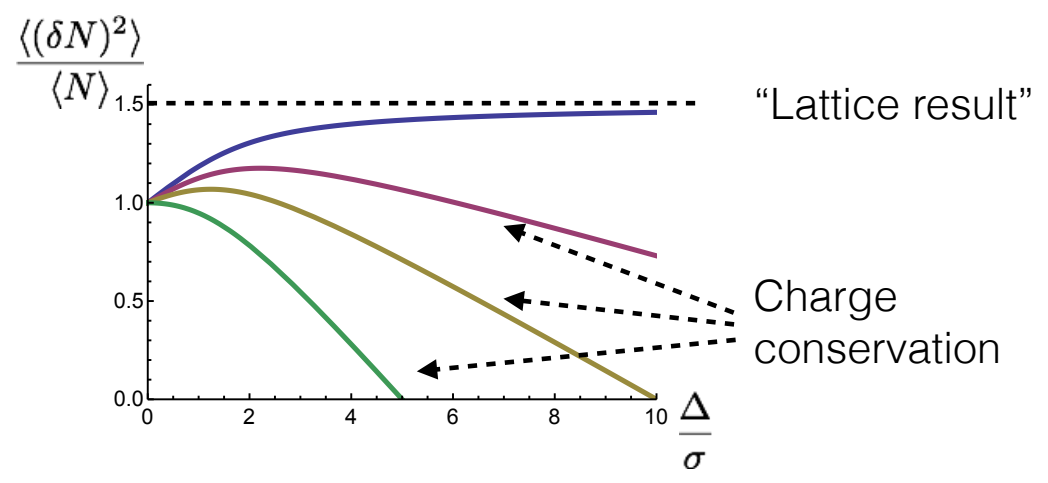

Figure 2: Observed variance as a function of acceptance. The black dashed line gives the infinite volume/acceptance limit which will also be what lattice QCD will calculate. The blue line give the value without charge conservation effects, while the magenta, yellow, and green line represent the measured values if the total charge is conserved over a acceptance interval of $\Delta_{\text {charge }} / \sigma=5,10,20$, respectively. Here $\sigma$ is the correlation length.

The main lesson from this simple example, as well as from the observed acceptance dependence reported by STAR, is that any comparison of lattice QCD and other model calculation with measured cumulant ratio needs to be done with extreme care. Effects from global charge conservation need to be removed, and the cumulant ratio has to be measured over a sufficiently wide range of acceptance to allow for an extrapolation to infinite acceptance. Only then can we compare with theoretical calculations in the infinite volume limit. As an example, where such an extrapolation has been attempted, we refer to the extraction of the net charge fluctuations by the ALICE collaboration [22]. Recent comparisons of cumulant ratios obtained, by lattice QCD with the STAR measurements in order to extract freeze out parameters [23, 24], need to be viewed in this light. Of 
course, if the correlations are weak and effects of charge conservation are small, as is the case for collisions at the highest energies, the above considerations are not relevant. This may very well be the reason why the freeze out parameters extracted from lattice agree rather well with those from an analysis based on an uncorrelated hadron resonance gas [25].

\section{The first order phase co-existence region}

Let us next turn to the first order co-existence region. While the discussion concerning the QCD phase structure focuses on the critical point, it is worthwhile to discuss observable effects due to the first order co-existence region. From the phenomenological point of view there is no a priori difference between the discovery of the critical point and that of the first order phase coexistence region. Both would clearly demonstrate the existence of a non-trivial structure in the QCD phase diagram.

One feature of a first order transition, which may be useful in the context of heavy ion collisions, is the presence of the so-called spinodal region, which is characterized by mechanical instability, $\partial p / \partial \varepsilon<0$. This instability leads to exponential growth of local density fluctuations and a fast phase separation into blobs of high density and low density matter of characteristic size, which is governed by the surface tension. The spinodal region lies in the thermodynamically unstable region of the phase diagram and can only be accessed by a dynamical, non-adiabatic process such as a heavy ion collision. In addition, the fast exponential growth of the density fluctuations increases the likelihood for an observable signal to be produced during the rather short lifetime of the system. This is to be contrasted to the critical slowing down, which inhibits the growth of fluctuations in the vicinity of a second order transition, such as the CP [26]. Recently a first study of the effect of spinodal separation has been carried out using hydrodynamics $[27,28,29,30]$. In
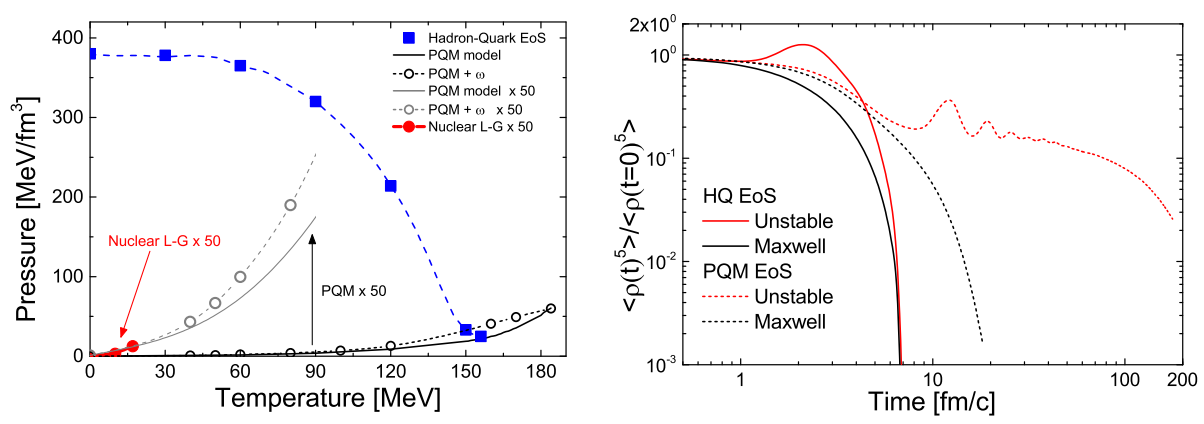

Figure 3: Left: Phase diagram for the Polyakov-quark-meson model together with a parametrization of a possible QCD transition, where the co-existence pressure at vanishing temperature has been chosen to be considerably higher than the pressure at $(T, \mu)=\left(T_{p c}, 0\right)$. Also shown is the nuclear liquid-gas co-existence line. Right: The fifth moment of the density distribution as a function of time in a hydrodynamic evolution for the two equations of state of the left panel. For details see [30] where the figures are adapted from.

[29] the authors found that, due to the spinodal instability initial state density, fluctuations were considerably enhanced. This can be seen in the right panel of Fig. 3, where we show the time evolution of the fifth moment of the density distribution for two equations of state with (red lines) 
and without (black lines) spinodal instabilities. In the left panel we show the two equations of state which have been used in this study [30]. The blue line represents a "QCD inspired" EoS with large co-existence pressure at vanishing temperature, whereas the black line is a liquid-gas type equation of state which is typically obtained in effective mean field models such as the Polyakov quark meson model, which has been used here. The effect of the equation of state on the time evolution of the density fluctuation is significant, as can be seen by comparing the full and dashed red line in the right panel. In both cases we see significant enhancement of density fluctuations, which for the liquid-gas type equation of state last nearly $100 \mathrm{fm} / \mathrm{c}$ ! This is simply the result of the small co-existence pressure inherent in a liquid-gas transition. Nearly stable blobs of QGP are being formed, which oscillate before they slowly evaporate. In contrast, in case of the "QCD" equation of state the enhanced density fluctuations die out rather quickly. The large co-existence pressure leads to an "explosion" of the blobs once the surrounding nuclear matter, which counterbalances the high pressure of the dense phase, has expanded away.

Given the sizable enhancement of the moments of the baryon density exhibited in Fig. 3, one would naively expect that this should be observable in an enhanced productions of small nuclei such as ${ }^{4} \mathrm{He}$ or ${ }^{7} \mathrm{Li}$ etc. This idea has also been investigated in [30] with the result that the enhancement is quite moderate, of the order of $20 \%$. The reason is the aforementioned entropy to baryon-number ratio which in case of the "QCD" transition is larger in the QGP phase then in the hadron phase. Since the formation of nuclei or clusters is usually governed by phase space coalescence, the large entropy per baryon in the dense phase inhibits the formation of clusters. While the baryon number is closely packed in coordinate space, as evidenced by the large density moments, the large entropy per baryon leads to a dilution in momentum space so that the resulting phase space density, and thus the formation of clusters, is only mildly enhanced.

\section{Summary}

In this contribution we have discussed some general properties of the QCD phase diagram. We have pointed out that, contrary to a liquid-gas transition, the QCD transition has a finite and large co-existence pressure at zero temperature. We have noted that most effective models predicting a critical point are of the liquid-gas type, i.e. they predict a vanishing co-existence pressure, limiting their phenomenological usefulness. We further discussed the dynamical consequences of the large co-existence pressure in the framework of hydrodynamics. The blobs formed as result of the spinodal breakup decay very rapidly making a detection rather difficult. We also stressed a few caveats which need to be addressed when comparing lattice and model calculations with measured cumulant ratios. Since theoretical calculations are typically carried out in the infinite volume limit and in the grand canonical ensemble, one needs to correct the data for effects due to global charge conservation. In addition, one has to analyze the cumulants for various acceptance windows in order to allow an extrapolation to infinite acceptance. Only then is comparison with theory meaningful.

To summarize, there has been considerable progress in the search for the QCD phase structure. The new, preliminary results on the net-proton kurtosis by the STAR collaboration [17] are very exciting and are qualitatively consistent with expectations for fluctuations near a critical point [14]. However, better data with smaller error bars together with a more comprehensive theoreti- 
cal framework, which addresses also background contributions such as stopping fluctuations in a quantitative way, are needed before we can declare success.

\section{Acknowledgments}

This work was supported by the Office of Nuclear Physics in the US Department of Energy's Office of Science under Contract No. DE-AC02-05CH11231.

\section{References}

[1] Y. Aoki, G. Endrodi, Z. Fodor, S. D. Katz, and K. K. Szabo Nature 443 (2006) 675-678, arXiv:hep-lat/0611014.

[2] A. Bazavov, T. Bhattacharya, M. Cheng, C. DeTar, H. Ding, F. Karsch, et al. Phys.Rev. D85 (2012) 054503, arXiv:1111.1710 [hep-lat].

[3] Y. Aoki, Z. Fodor, S. D. Katz, and K. K. Szabo Phys. Lett. B643 (2006) 46-54, arXiv:hep-lat/0609068.

[4] Y. Aoki, S. Borsanyi, S. Durr, Z. Fodor, S. D. Katz, et al. JHEP 0906 (2009) 088, arXiv:0903.4155 [hep-lat].

[5] HotQCD Collaboration, A. Bazavov et al. Phys.Rev. D90 (2014) 094503, arXiv: 1407.6387 [hep-lat].

[6] M. Stephanov PoS LAT2006 (2006) 024, arXiv: hep-lat/ 0701002 [hep-lat] .

[7] Z. Fodor and S. Katz JHEP 0404 (2004) 050, arXiv: hep-lat/0402006 [hep-lat ].

[8] R. Gavai and S. Gupta Phys.Rev. D78 (2008) 114503, arXiv:0806.2233 [hep-lat ].

[9] S. Datta, R. V. Gavai, and S. Gupta Nucl.Phys. A904-905 (2013) 883c-886c, arXiv:1210 . 6784 [hep-lat].

[10] M. Prakash, T. Ainsworth, and J. Lattimer Phys.Rev.Lett. 61 (1988) 2518-2521.

[11] M. Prakash, Lecture Notes of the workshop on the The Nuclear Equation of State, Jan 4 - 14, 1994, Puri, India, A. Ansari and L. Satpathy (eds), (World Scientific, 1996) pp: 229-410.

[12] M. Hempel, V. Dexheimer, S. Schramm, and I. Iosilevskiy Phys.Rev. C88 no. 1, (2013) 014906, arXiv:1302.2835 [nucl-th].

[13] M. Stephanov Phys.Rev.Lett. 102 (2009) 032301, arXiv:0809.3450 [hep-ph].

[14] M. Stephanov Phys.Rev.Lett. 107 (2011) 052301, arXiv:1104.1627 [hep-ph].

[15] M. A. Stephanov, K. Rajagopal, and E. V. Shuryak Phys.Rev. D60 (1999) 114028, arXiv:hep-ph/9903292 [hep-ph].

[16] STAR Collaboration Collaboration, L. Adamczyk et al. arXiv:1309.5681 [nucl-ex] .

[17] STAR Collaboration, X. Luo PoS CPOD2014 (2014) 019, arXiv:1503.02558 [nucl-ex].

[18] A. Bzdak and V. Koch Phys.Rev. C86 (2012) 044904, arXiv:1206.4286 [nucl-th].

[19] A. Bzdak, V. Koch, and V. Skokov Phys.Rev. C87 (2013) 014901, arXiv:1203. 4529 [hep-ph]. 
[20] M. Bleicher, S. Jeon, and V. Koch Phys.Rev. C62 (2000) 061902, arXiv: hep-ph/ 0006201 [hep-ph].

[21] M. Sakaida, M. Asakawa, and M. Kitazawa Phys.Rev. C90 no. 6, (2014) 064911, arXiv:1409.6866 [nucl-th].

[22] ALICE Collaboration, B. Abelev et al. Phys.Rev.Lett. 110 no. 15, (2013) 152301, arXiv:1207.6068 [nucl-ex].

[23] A. Bazavov, H. Ding, P. Hegde, O. Kaczmarek, F. Karsch, et al. Phys.Rev.Lett. 109 (2012) 192302, arXiv:1208.1220 [hep-lat].

[24] S. Borsanyi, Z. Fodor, S. Katz, S. Krieg, C. Ratti, et al. Phys.Rev.Lett. 113 (2014) 052301, arXiv:1403.4576 [hep-lat].

[25] A. Andronic Int.J.Mod.Phys. A29 (2014) 1430047, arXiv:1407.5003 [nucl-ex].

[26] B. Berdnikov and K. Rajagopal Phys.Rev. D61 (2000) 105017, arXiv:hep-ph/9912274 [hep-ph] .

[27] J. Randrup Phys. Rev. Lett. 92 (2004) 122301, arXiv: hep-ph/0308271.

[28] J. Randrup Phys. Rev. C79 (2009) 054911, arXiv:0903.4736 [nucl-th].

[29] J. Steinheimer and J. Randrup Phys.Rev.Lett. 109 (2012) 212301, arXiv : 1209.2462 [nucl-th].

[30] J. Steinheimer, J. Randrup, and V. Koch Phys.Rev. C89 (2014) 034901, arXiv:1311. 0999 $[$ nucl-th]. 